\title{
Adaptive Control of a Simple Nonlinear System without a priori Information on the Plant Parameters
}

\author{
Rogelio Lozano and Bernard Brogliato
}

\begin{abstract}
This paper presents an adaptive control scheme for nonlinear systems of the form $\dot{x}=c^{* T} f(x)+b^{*} u$, where $f(x)$ is Lipschitz, $c^{*}$ is a constant vector, and $b^{*}$ is a constant scalar. The control scheme achieves asymptotical model matching without a priori knowledge of the sign of the $b^{*}$ gain. The adaptive scheme is free from singularities in the sense that the estimate of $b^{*}$, entering in the denominator of the control law, is bounded away from zero. The singularity has been overcome through a suitable modification of the parameters estimates which is based on standard least squares covariance matrix properties.
\end{abstract}

\section{INTRODUCTION}

A DAPTIVE control of linear continuous-time systems has been successfully developed in the last decade [1]- [3]. Nevertheless it is only recently that adaptive systems techniques have been considered to control nonlinear systems [4]-[6]. These results represent contributions to the field and open the way to the application of adaptive control to nonlinear plants. However, there still exist some problems that have not been clearly solved in the linear case and are even more complex in the nonlinear case. In particular, the problem associated to the a priori knowledge of the highfrequency gain raised in [7], has not received a satisfactory answer and deserves special attention in the nonlinear case. It is clear that there exist particular cases for which the sign of the frequency gain can be known a priori. Furthermore, if the plant is stable and, provided we can introduce high-frequency probing signals into the system, then the high-frequency gain can be identified off-line before the adaptive control is applied to the system. However, the assumption on the knowledge of the sign of the high-frequency gain does not appear to be realistic in the general case.

One of the first adaptive control algorithms for nonlinear systems was presented in [5]. Nevertheless this control scheme is not free from eventual singularities. Indeed the control law's denominator is a function of the parameters estimates and has not been ensured to be bounded away from zero.

The first adaptive control scheme for linear systems not

Manuscript received March 2, 1990; revised July 20, 1990 and March 8. 1991. Paper recommended by Associate Editor. Technical Notes and Correspondence, J. Baillieul.

R. Lozano is with UTC-HEUDIASYC, BP 649-60206 Compiègne, France. B. Brogliato is with L.A.G.-E.N.S.I.E.G., BP 46-38402 Saint M. d'Hères, France.

IEEE Log Number 9104611 using the a priori knowledge of the sign of the high-frequency gain was presented in [8]. This technique was generalized in [9] for linear systems, and was used in [6] to develop a control scheme for first-order nonlinear systems. However, the main drawback of schemes based on the Nussbaum's gain in [8] is that the transient behavior is quite violent.

An alternative way to solve the problem was presented in [10] based on a particular modification of the parameters estimates to avoid division by zero. The technique follows the work in [11] for discrete-time systems, however, the problem of existence of solutions that arises naturally in continuous-time adaptive control has not been addressed.

In [12] it is shown that if one uses an adequate discontinuous control, similar to the one proposed in [10] and [11], then existence of the solutions is ensured and all the signals remain bounded. The proof of boundedness was carried out assuming that the time derivative of the output was available to the parameter estimator and current research is under way to extend the result.

In this paper, we present an adaptive control for nonlinear systems of the form $\dot{x}=c^{* T} f(x)+b^{*} u$, where $f(x)$ is Lipschitz, $c^{*}$ is a constant vector, and $b^{*}$ is a constant scalar. The control scheme is shown to achieve asymptotical model matching and keep all the signals bounded without requiring knowledge of the sign of the $b^{*}$ gain. Loss of stabilizability of the estimated model is avoided by appropriately modifying the parameters estimates. The proposed modification is based on the least squares covariance matrix and is done in such a way that the modified estimates preserve the essential properties of the original estimates. Furthermore, the analysis establishes existence of solutions of the various ordinary differential equations involved in the closed-loop control scheme following the ideas in [13] and [14]. A short preliminary version of the present paper was presented in [15] but unfortunately the problem of existence of solutions was not considered at that time.

The outline of the paper is as follows. Section II presents the problem formulation and the developments to obtain the error equation. The parameters estimation algorithm and the estimates modification procedure are described in Section III. Section IV presents the full set of ordinary differential equations involved in the closed-loop adaptive control system. Section V is devoted to show the existence of the solutions and some properties of the proposed estimation algorithm. The rest of the convergence analysis is found in Section VI. The conclusions are finally given in Section VII. 


\section{Problem Formulation}

Consider the class of nonlinear systems

$$
\dot{x}=c^{* T} f(x)+b^{*} u
$$

where $x, u \in \mathbb{R}, b^{*}$ is a constant scalar, and $c^{*}$ is a constant vector. We make the following assumptions on the system.

\section{Assumptions}

$A 1: b^{*} \neq 0$.

$A 2: f(x)$ is a known Lipschitz vector function satisfying the sector condition

$$
\|f(x)\| \leq k|x|+k \quad \text { for some } k>0 .
$$

The desired state trajectory is given by the model

$$
\dot{x}_{m}=-a x_{m}+r
$$

where $a>0$ and $r$ is a bounded reference input.

Define the tracking error as

therefore

$$
e=x-x_{m}
$$

$$
\dot{e}=-a e-\theta^{* T} \phi+a x-r
$$

where

$$
\begin{gathered}
\theta^{*^{T}}=\left[b^{*}, c^{*^{T}}\right] \\
\phi^{T}=\left[-u,-f(x)^{T}\right] .
\end{gathered}
$$

Equation (5) can also be written as

$$
e=h *\left(-\theta^{* T} \phi+a x-r\right)
$$

where $h * w$ denotes the convolution between $h$ and $w$, and $h$ is the inverse Laplace transform of $H(s)$ given by

$$
H(s)=\frac{1}{s+a} .
$$

Since $H(s)$ is an asymptotically stable transfer function, it is clear from (8) that if the control input is such that

$$
-\theta^{* T} \phi+a x-r=0
$$

or equivalently

$$
u=\frac{-c^{* T} f(x)-a x+r}{b^{*}}
$$

then the tracking error $e$ will converge to zero. When the parameters vector $\theta^{*}$ is unknown, it is standard to replace it by its estimate $\theta$ to obtain an adaptive control scheme. The parameters estimates vector $\theta$ is decomposed as $\theta^{*}$ in (6), i.e.,

$$
\theta^{T}=\left[b, c^{T}\right] .
$$

Nevertheless, $b$ in $\theta$ may approach zero and consequently such an adaptive control law would not be implementable. Therefore, instead of using directly the current parameters estimates $\theta$ we propose to appropriately modify them before using them in the adaptive control law to avoid any singularities. The modified estimates vector will be denoted by $\bar{\theta}$ and is also decomposed as

$$
\bar{\theta}=\left[\bar{b}, \bar{c}^{T}\right] .
$$

The adaptive control law using the modified parameter estimates is given by

$$
u=\frac{-\bar{c}^{T} f(x)-a x+r}{\bar{b}}
$$

or equivalently

$$
-\bar{\theta}^{T} \phi+a x-r=0 .
$$

The modified parameters are given by

$$
\bar{\theta}=\theta+P \alpha
$$

where $\theta$ is the estimates vector, $P$ is the covariance matrix, and $\alpha$ is the correction vector in Fig. 1 that will be defined in the next section.

\section{Augmented Error Equation}

Introducing the adaptive control law (13)' into the equation error (8) we obtain

$$
e=h *\left(\left(\bar{\theta}-\theta^{*}\right)^{T} \phi\right) .
$$

Introducing (14) into (15) we obtain

$$
e=h *\left(\tilde{\theta}^{T} \phi+\alpha^{T} P \phi\right)
$$

where

$$
\tilde{\theta}=\theta-\theta^{*} .
$$

Let us now define the following augmented error

$$
e_{a}=e-h *\left(\alpha^{T} P \phi\right)+\theta^{T} h *(\phi)-h *\left(\theta^{T} \phi\right) .
$$

Since the constant $\theta^{*}$ commutes with convolution

$$
e_{a}=e-h *\left(\alpha^{T} P \phi\right)+\tilde{\theta}^{T} h *(\phi)-h *\left(\tilde{\theta}^{T} \phi\right) .
$$

Introducing (16) into the above equation, we finally obtain

$$
e_{a}=\tilde{\theta}^{T} \xi
$$

where

$$
\xi=h *(\phi) .
$$

\section{Parameters Estimation and Correction Procedure}

In order to complete the adaptive control design, we define next the parameters estimates vector $\theta$, the covariance matrix $P$, and the correction vector $\alpha$ that were mentioned in the previous section.

As parameter adjustment law we will use the following normalized least squares (LS) algorithm

$$
\begin{gathered}
\dot{\theta}=-\frac{P \xi}{m^{2}} e_{a} \\
\dot{P}=-\frac{P \xi \xi^{T} P}{m^{2}} ; \quad P(0)>0 \\
m=\max _{t}(1+|x|) .
\end{gathered}
$$




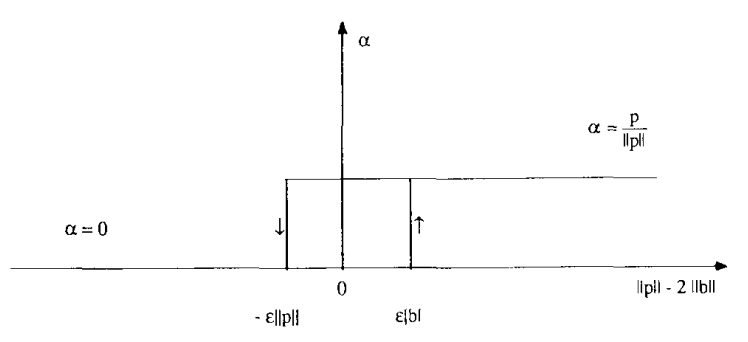

Fig. 1. Parameters estimates modification procedure.

\section{Parameters Estimates Modification}

In order to avoid any possible singularities in the adaptive control we propose to modify the estimates as in (14) with $\alpha$ as in Fig. 1. As will appear clear later, the hysteresis in $\alpha$ is introduced to ensure that the modified parameters vector $\bar{\theta}$ converge and to prevent the discontinuities in $\alpha$ from occurring an infinite number of times or infinitely often. The correction vector $\alpha$ in Fig. 1 was first used in [16]. In Fig. 1, $p^{T}$ denotes the first row of $P, b$ the first element of $\theta$, and $\epsilon$ is an arbitrary constant such that $0<\epsilon \ll 1$

$$
\begin{gathered}
p^{T}=\left[\begin{array}{cccc}
1 & 0 & \cdots & 0
\end{array}\right] P \\
0<\epsilon \ll 1 .
\end{gathered}
$$

\section{The Reasoning Behind the Estimates Modification}

The parameters estimates modification described in (14) and Fig. 1 is based on the following two properties of least squares estimation algorithms. First, that under certain conditions, adding a vector on the image of the covariance matrix $P$ to the current estimates, as in (14), does not change the basic convergence properties of the original estimates. This will be shown in Section VI. Second, that the estimate $b$ and its corresponding row in $P$, i.e., $p$, never vanish simultaneously. This is shown in Lemma 2 . Therefore, $\alpha$ in Fig. 1 is chosen equal to 0 when $b$ is larger than a certain fraction of $\|p\|$, otherwise is chosen equal to $p /\|p\|$. This choice of $\alpha$ guarantees a lower bound for $|\bar{b}|$ as is established in Lemma 2. The hysteresis in Fig. 1 is introduced to avoid that the discontinuities occur infinitely often and also to guarantee that the modified estimates converge.

\section{Closed-Loop System State-Space REPRESENTATION}

The adaptive control scheme presented in the previous sections is unconventional in adaptive systems because it involves nonlinearities and discontinuities. Therefore the question of existence and uniqueness of the solutions of the overall nonlinear system has to be examined. For that purpose this section presents the complete closed-loop system state-space representation.

Introducing the adaptive control law (13) into the system (1) we obtain

$$
\dot{x}=c^{* T} f(x)+b^{*}\left[\frac{-\bar{c}^{T} f(x)-a x+r}{\bar{b}}\right] .
$$

Equation (18) can also be rewritten as (see also (4), (9), and (21))

$$
e_{a}=x-x_{m}-z_{1}+\theta^{T} \xi-z_{2}
$$

where $z_{1}$ and $z_{2}$ are defined below and $\xi$ in (21) can also be written as follows

$$
\begin{gathered}
\dot{z}_{1}=-a z_{1}+\alpha^{T} P \phi \\
\dot{z}_{2}=-a z_{2}+\theta^{T} \phi \\
\dot{\xi}=-a \xi+\phi
\end{gathered}
$$

where $\phi$ is given in (7) or, introducing (13)

$$
\phi^{T}=\left[\frac{\bar{c}^{T} f(x)+a x-r}{\bar{b}},-f(x)^{T}\right] .
$$

Therefore the full set of ordinary differential equations involved in the closed-loop system are (see (3), (22), (23), (27), (29)-(31))

$$
\left.\begin{array}{l}
\dot{x}=c^{* T} f(x)+b^{*}\left[\frac{-\bar{c}^{T} f(x)-a x+r}{\bar{b}}\right] \\
\dot{x}_{m}=-a x_{m}+r \\
\dot{z}_{1}=-a z_{1}+\alpha^{T} P \phi \\
\dot{z}_{2}=-a z_{2}+\theta^{T} \phi \\
\dot{\xi}=-a \xi+\phi \\
\dot{\theta}=-\frac{P \xi}{m^{2}} e_{a} \\
\dot{P}=-\frac{P \xi \xi^{T} P}{m^{2}}
\end{array}\right\}
$$

with $\bar{\theta}^{T}=\left[\bar{b}, \bar{c}^{T}\right]$ in (14), $\phi$ in (32), $e_{a}$ in (28), $m$ in (24) and $\alpha$ in Fig. 1.

\section{Existence and Uniqueness of SOlutions}

In this section, we present the convergence properties of the parameters estimation algorithm and those of the estimates modification procedure proposed in the previous sections. These properties allow us to establish the existence and uniqueness of solutions of the ordinary differential equations (33) involved in the closed-loop control system. These results are a prerequisite to the convergence analysis given in the next section.

Let us denote by $x_{\mathrm{cl}}$ the state of entire closed-loop control system (33). Thus $x_{\mathrm{cl}}$ is formed by the augmentation of the states $x, x_{m}, z_{1}, z_{2}, \xi, \theta$ and the rows of $P$.

Definition: Let us define $D_{1}=\left[0, t_{1}\right) \times B$ as the bounded domain for $\left\{t, x_{\mathrm{cl}}\right\}$ where $0 \leq t<t_{1}$ and $x_{\mathrm{cl}} \in B$, for some open connected set $B \in \mathscr{R}^{n}$. The time $t_{1}$ is defined in such a 
way that $\alpha$ presents no discontinuities in the interval $\left[0, t_{1}\right)$ (i.e., $\alpha$ remains either equal to 0 or equal to $p /\|p\|$ ) and also such that $\bar{b}$ is bounded away from zero in $\left[0, t_{1}\right)$.

Lemma 1: The closed-loop control system differential equations (33) have a solution on the bounded domain $D_{1}$.

Proof: Examination of the differential equations (33) reveals that the main source of discontinuities is $\alpha$ (or $\bar{\theta}$ ). Since $m$ in (24) is nonsingular, it does not introduce any undesirable effects on (33). In view of the definition of the bounded domain $D_{1}$ it follows that the RHS of (33) is Lipschitz in $D_{1}$ (see [13], [14], and [17]). Therefore, for any initial condition in $D_{1}$, a unique solution exists for $0 \leq$ $t<t_{1}$.

The following lemma establishes that the estimates modification procedure defined in Fig. 1 is such that, along the solutions in the domain $D_{1}$, the hysteresis width in $\alpha$ and $\bar{b}$ remain bounded away from zero.

Lemma 2: The parameters estimation algorithm and the estimates correction procedure defined in Section III are such that the following results hold along the solutions in $D_{1}=$ $\left[0, t_{1}\right) \times B$. Furthermore, the results hold independently of the size of $B$.

$$
\begin{aligned}
& \text { 1) } P^{-1} \tilde{\theta}=P^{-1}(0) \tilde{\theta}(0) \\
& \text { 2) }|b|+\|p\| \geq b^{\prime}
\end{aligned}
$$

with

$$
b^{\prime}=\left|b^{*}\right| / \max \left(1,\left\|P^{-1}(0) \tilde{\theta}(0)\right\|\right)
$$

3) $|\bar{b}| \geq \frac{1-\epsilon}{3+\epsilon} b^{\prime}$.

Proof:

1) Derivating the expression $P P^{-1}=I$ one obtains

$$
\frac{d P^{-1}}{d t}=-P^{-1} \dot{P} P^{-1} .
$$

Introducing (23) we get

$$
\frac{d P^{-1}}{d t}=\frac{\xi \xi^{T}}{m^{2}} .
$$

Using (17), (22), and the above, it follows that

$$
\frac{d\left(P^{-1} \tilde{\theta}\right)}{d t}=\frac{\xi \xi^{T}}{m^{2}} \tilde{\theta}-\frac{\xi}{m^{2}} e_{a} .
$$

Introducing (20) in the above we finally obtain

$$
\frac{d\left(P^{-1} \tilde{\theta}\right)}{d t}=0
$$

from which (34) follows.

2) Equation (34) can also be rewritten as

$$
\tilde{\theta}=P P^{-1}(0) \tilde{\theta}(0) \text {. }
$$

Premultiplying the above by $[100 \cdots 00]$ we obtain (see also (6), (11), (17), and (25))

$$
b-b^{*}=p^{T} P^{-1}(0) \tilde{\theta}(0) \text {. }
$$

We then have

$$
\begin{aligned}
\left|b^{*}\right| & =\left|b-p^{T} P^{-1}(0) \tilde{\theta}(0)\right| \\
& \leq|b|+\|p\|\left\|P^{-1}(0) \tilde{\theta}(0)\right\| \\
& \leq(|b|+\|p\|) \max \left(1,\left\|P^{-1}(0) \tilde{\theta}(0)\right\|\right)
\end{aligned}
$$

from which (35) follows.

3 ) We will study separately the only two possible cases.

Case $\alpha=0$ : From (14) we have

$$
\bar{b}=b \text {. }
$$

From Fig. 1

$$
\|p\|-2|b| \leq \epsilon|b|
$$

or

$$
\|p\| \leq(2+\epsilon)|b| .
$$

Introducing (42) and (43) into (35)

$$
b^{\prime} \leq|b|+\|p\| \leq(3+\epsilon)|b|=(3+\epsilon)|\bar{b}| .
$$

Therefore (37) is verified in this case.

Case $\alpha=p /\|p\|$ : From Fig. 1

$$
\|p\|-2|b| \geq-\epsilon\|p\| \text {. }
$$

From (14) we have (see also (11), (12), and (25))

$$
\vec{b}=b+p^{T} p /\|p\| \text {. }
$$

Therefore

$$
\begin{aligned}
|\bar{b}| & =|b+\|p\|| \\
& \geq\|p\|-|b| \\
& =\frac{1}{2}\|p\|+\frac{1}{2}[\|p\|-2\|b\|] \\
& \geq \frac{1}{2}\|p\|-\frac{1}{2} \epsilon\|p\|(\operatorname{using}(45)) \\
& =\frac{1-\epsilon}{2}\|p\| .
\end{aligned}
$$

Combining (35) and (45)

$$
\begin{aligned}
b^{\prime} & \leq|b|+\|p\| \\
& \leq \frac{1+\epsilon}{2}\|p\|+\|p\|=\frac{3+\epsilon}{2}\|p\| .
\end{aligned}
$$

Introducing (47) into (46) we obtain

$$
|\bar{b}| \geq \frac{1-\epsilon}{2}\|p\| \geq \frac{1-\epsilon}{3+\epsilon} b^{\prime}
$$

which concludes the proof.

In view of Lemma 2, the result in Lemma 1 can be extended to hold in a larger domain which is established next.

Definition: Let us now define $D=[0, T) \times B$ as the bounded domain for $\left\{T, x_{\mathrm{cl}}\right\}$ where $T \in \mathscr{R}^{+}$and $x_{\mathrm{cl}} \in B$, for some open connected set $B \in \mathscr{R}^{n}$.

Lemma 3: The closed-loop control system differential equations (33) have a solution on the bounded domain $D=$ $[0, T) \times B$.

Proof: Lemma 1 establishes the existence of the solu- 
tions of (33) in $D_{1}$. That means that the solution exists as long as $x_{\mathrm{cl}} \in B, \bar{b}$ is bounded away from zero and $\alpha$ present no discontinuities. On the other hand, Lemma 2 shows that $\bar{b}$ remains bounded away from zero (see (37)) along the solutions in $D_{1}$. We conclude then that the solution exists as long as $x_{\mathrm{cl}} \in B$ and $\alpha$ present no discontinuities. Lemma 2 also shows that the hysteresis width of $\alpha$ in Fig. 1 is bounded away from zero (see (35)). Thus, as long as $x_{\mathrm{cl}} \in B$, the time between two consecutive discontinuities in $\alpha$ will be clearly different from zero. This allows us to define a set of solutions in $D_{1}$ : the first solution starts at the initial condition and ends when the first discontinuity in $\alpha$ occur, the second solution starts at the final value of the first solution and ends when the second discontinuity occur, and so on. The RHS of (33) is piecewise continuous in $D$ and we can define a solution in the bounded domain $D$ as the concatenation of the solutions in $D_{1}$ defined in between those time instants where discontinuities in $\alpha$ occur. The solution can be extended to the boundary of $B$, i.e., the existence of solutions of (33) is guaranteed as long as $x_{\mathrm{cl}}$ is in $B$.

The following lemma establishes boundedness of the parameters estimates $\theta$ and the covariance matrix $P$ in the domain $D=[0, T) \times B$ independently of the size of $B$. This will allow us later to extend the solutions to a larger domain.

Lemma 4: The parameters estimates $\theta$ and the covariance matrix $P$ remain bounded along the solutions of the differential equations (33) in the domain $D=[0, T) \times B$ independently of the size of $B$, i.e.,

$$
\begin{array}{ll}
\text { 1) } 0<P \leq P(0) & 0<t \leq T \\
\text { 2) }\|\tilde{\theta}\| \leq\|P(0)\|\left\|P^{-1}(0) \tilde{\theta}(0)\right\| & 0<t \leq T .
\end{array}
$$

\section{Proof:}

1) Integrating (39)

$$
P^{-1}=P^{-1}(0)+\int_{0}^{t} \frac{\xi \xi^{T}}{m^{2}} d \tau ; \quad 0<t \leq T .
$$

Since $P^{-1}(0)>0$, it follows that $P^{-1}>0$ and thus $P>0$ for $0<t \leq T$. Integrating now (23) we obtain

$$
\int_{0}^{t} \frac{P \xi \xi^{T} P}{m^{2}} d \tau=P(0)-P \geq 0 ; \quad 0<t \leq T .
$$

From the above it then follows that $P \leq P(0)$.

2) From (34)

$$
\tilde{\theta}=P P^{-1}(0) \tilde{\theta}(0)
$$

Then

$$
\|\tilde{\theta}\| \leq\|P\|\left\|P^{-1}(0) \tilde{\theta}(0)\right\| \quad 0<t \leq T .
$$

It is shown in the Appendix that $P \leq P(0)$ (48) implies that $\|P\| \leq\|P(0)\|$ where $\|P\|$ denotes the euclidean norm of $P$. Therefore, (49) follows from the above equation.

The results in Lemma 4 will finally enable us to establish the existence of solutions for any time in $\mathscr{R}^{+}$as stated in the next Lemma.

Lemma 5: The closed-loop control system differential equations (33) have a solution over the interval $[0, T)$ with $T \in \mathscr{R}^{+}$. Furthermore, the state $x_{\mathrm{cl}}$ of the closed-loop system (33) does not grow faster than exponentially.

Proof: Lemma 3 shows that the differential equations (33) have a solution on the bounded domain $D=[0, T) \times B$. On the other hand, Lemma 4 establishes that $\theta$ and $P$ in (33) remain bounded independently of the size of the open connected set $B$. Since $\theta$ and $P$ are bounded, let us now consider only the reduced state $w^{T}=\left[x, x_{m}, z_{1}, z_{2}, \xi^{T}\right]$ in (33). Since by assumption A2, $f(x)$ is Lipschitz, it follows that the dynamic equations corresponding to $w$ have a RHS that is Lipschitz (see (33)) along the solutions in $D$. Therefore, $w$ does not grow faster than exponentially in $D$. Furthermore, since the bounds on $\theta$ and $P$ are independent of the size of $B$, the set $B$ can be chosen arbitrarily large and it follows then that $w$ does not grow faster than exponentially in a domain $D=[0, T) \times B$ with $B$ arbitrarily large. Therefore the solutions of (33) exists for $0 \leq t<\infty$ and $w$ does not grow faster than exponentially in $0 \leq t<\infty$.

\section{Vi. Convergence Analysis}

Once the existence of the solutions of the differential equations (33) has been established, we can complete the convergence analysis of the adaptive control presented in the previous sections. Some of the properties of the parameter estimation algorithm were presented in the previous section and the rest of the properties required to proceed with the analysis are given in the following Lemma.

Lemma 6: The adaptive control scheme presented in Sections II and III is such that the following properties hold:

1) $\frac{e_{a}}{m} \in L_{2}$

2) $\frac{P \xi}{m} \in L_{2}$

3) $P$ and $\theta$ converge

4) $\bar{\theta}$ converge and the discontinuities in $\alpha$ occur only a finite number of times.

Proof:

1) Consider the following positive definite function

$$
V=\frac{1}{2} \tilde{\theta}^{T} P^{-1} \tilde{\theta}
$$

Derivating

$$
\begin{aligned}
\dot{V} & =\frac{1}{2} \frac{d\left(\tilde{\theta}^{T}\right)}{d t} P^{-1} \tilde{\theta}+\frac{1}{2} \tilde{\theta}^{T} \frac{d\left(P^{-1} \tilde{\theta}\right)}{d t} \\
& \left.=\frac{1}{2} \frac{d\left(\tilde{\theta}^{T}\right)}{d t} P^{-1} \tilde{\theta} \quad \text { (using }(40)\right) \\
& =-\frac{1}{2} \frac{e_{a}^{2}}{m^{2}} .
\end{aligned}
$$

Integrating the above equation gives the desired result

$$
\int_{0}^{t} \frac{e_{a}^{2}}{m^{2}} d \tau=2(V(0)-V) \leq 2 V(0) .
$$


2) The result is obtained by rewriting (51) as follows:

$$
\int_{0}^{t} \frac{P \xi \xi^{T} P}{m^{2}} d \tau=P(0)-P \leq P(0) .
$$

3) The integral in the above equation is bounded and the integrand is positive semidefinite, therefore the integral converges and so does $P$. Convergence of $\theta$ follows by rewriting (34) as

$$
\theta=\theta^{*}+P P^{-1}(0) \tilde{\theta}(0) .
$$

4) Since $P$ and $\theta$ converge then $\|p\|-2\|b\|$ in Fig. 1 converges which implies that $\alpha$ converges too. Convergence of $\bar{\theta}$ then follows from (14). Finally, since the hysteresis width in Fig. 1 is bounded away from zero (see (35)), it also follows that the total number of switches in $\alpha$ is finite.

We can finally state the final result.

Theorem: Consider the adaptive control presented in Sections II and III. Then the following properties are verified.

$$
\begin{aligned}
& \text { 1) } \frac{\phi}{m} \text { and } \frac{\xi}{m} \text { are bounded. } \\
& \text { 2) } \frac{e}{m} \in L_{2} \text {. }
\end{aligned}
$$

3) The tracking error $e$ converges to zero and all the variables remain bounded.

\section{Proof:}

1) Consider the control law (13). Since $f(x)$ satisfies (2), $r$ is bounded, $\bar{\theta}^{T}=\left[\bar{b}, \bar{c}^{T}\right]$ in (12) and (14) is bounded and $\bar{b}$ is bounded away from zero (see Lemmas 2 and 4 ), it follows that

$$
|u| \leq k|x|+k
$$

where $k$ denotes generically a positive real constant. From (2), (7), and the above we have

$$
\|\phi\| \leq k|x|+k .
$$

From the above and (24) it readily follows that $\phi / m$ is bounded. On the other hand, from (21) and the fact that $H(s)$ in (9) is asymptotically stable, we have

$$
\begin{aligned}
\|\xi\| & \leq k \max _{t}\|\phi\|+k \\
& \left.\leq k \max _{t}|x|+k \quad \text { (using }(60)\right) .
\end{aligned}
$$

From (24) and the above we conclude that $\|\xi\| / m$ is also bounded.

2) Equation (16) can also be rewritten as

$$
\begin{aligned}
e & =h *\left(\tilde{\theta}^{T} P^{-1} P \phi+\alpha^{T} P \phi\right) \\
& =h *\left(\tilde{\theta}(0)^{T} P(0)^{-1} P \phi+\alpha^{T} P \phi\right) \quad \text { (using (34)) }
\end{aligned}
$$

or

$$
e=h *\left(v^{T} P \phi\right)
$$

with

$$
v=\alpha+P(0)^{-1} \tilde{\theta}(0) .
$$

The swapping lemma for the stable filter $H(s)$ in (9) and any vectors $w$ and $z$ can be written as (see [18])

$$
h *\left(w^{T} z\right)-h *\left(w^{T}\right) z=-h *\left\{h *\left(w^{T}\right) \dot{z}\right\} .
$$

Introducing (63) into (61) with $w=\phi$ and $z=P v$ and using (21) we obtain

$$
\frac{e}{m}=\frac{\xi^{T} P v}{m}-\frac{h *\left(\xi^{T} \dot{P} v\right)}{m}-\frac{h *\left(\xi^{T} P \dot{\alpha}\right)}{m} .
$$

Note that in view of (53) and since $v$ in (62) is bounded it follows that

$$
\frac{\xi^{T} P v}{m} \in L_{2} .
$$

Let us now study the second term in the RHS of (64) which, using (23), can also be written as

$$
\frac{h *\left(\xi^{T} \dot{P} v\right)}{m}=\frac{1}{m} h *(m \beta)
$$

with

$$
\beta=-\frac{\xi^{T}}{m} \frac{P \xi}{m} \frac{\xi^{T} P}{m} v
$$

In view of (53) and (58) and since $P$ and $v$ are bounded it follows that $\beta \in L_{2} \cap L_{\infty}$. On the other hand, from (66) we have

$$
\begin{aligned}
\left|\frac{h *\left(\xi^{T} \dot{P} v\right)}{m}\right| & =\left|\frac{1}{m(t)}\left\{\epsilon(t)+\int_{0}^{t} e^{-a(t-\tau)} m(\tau) \beta(\tau) d \tau\right\}\right| \\
& \leq \frac{\epsilon}{m}+\int_{0}^{t} e^{-a(t-\tau)} \frac{m(\tau)}{m(t)}|\beta(\tau)| d \tau \\
& \leq \frac{\epsilon}{m}+\int_{0}^{t} e^{-a(t-\tau)}|\beta(\tau)| d \tau \quad \text { (using (24)) }
\end{aligned}
$$

where $\epsilon$ represents an exponentially decaying term due to initial conditions and therefore $\epsilon / m \in L_{2} \cap L_{\infty}$. Since $\beta \epsilon$ $L_{2} \cap L_{\infty}$, the RHS of (68) is $L_{2} \cap L_{\infty}$ (see [19, p. 59]).

Let us finally study the last term in the RHS of (64). Recall that the discontinuities in $\alpha$ occur only a finite number of times (see Lemma 6). Recall also that the signals do not grow faster than exponentially with time (see Lemma 5). Therefore, since $H(s)$ in (9) is Hurwitz, in this part of the analysis we only need to consider the two different values for $\alpha$ in the limit, i.e., $\alpha=0$ or $\alpha=p /\|p\|$. For $\alpha=0$ we have $\dot{\alpha}=0$ and thus the last term in (64) is $L_{2} \cap L_{\infty}$. For $\alpha=p /\|p\|, \dot{\alpha}$ is

$$
\dot{\alpha}=\frac{\dot{p}\|p\|^{2}-p p^{T} \dot{p}}{\|p\|^{3}} .
$$

From (23) and the above and since $p$ is bounded

$$
|\dot{\alpha}| \leq k\|\dot{p}\| \leq k\left\|\left[\begin{array}{llll}
1 & 0 & \cdots & 0
\end{array}\right] \frac{P \xi \xi^{T} P}{m^{2}}\right\|
$$


and thus the last term in (64) can also be expressed as

$$
\frac{h *\left(\xi^{T} P \dot{\alpha}\right)}{m}=\frac{1}{m} h *\left(m \beta^{\prime}\right)
$$

with

$$
\beta^{\prime}=\frac{\xi^{T} P}{m} \dot{\alpha}
$$

In view of (70), $\dot{\alpha} \in L_{2} \cap L_{\infty}$ and thus $\beta^{\prime} \in L_{2} \cap L_{\infty}$. Using a procedure similar to the one used for the second term in (64) we conclude that the last term in (64) is also $L_{2} \cap L_{\infty}$. Finally, it follows that $e / m$ in (64) is also $L_{2} \cap L_{\infty}$.

3 ) In order to prove that $e / m$ converges to zero we will first show that $e / m$ is Lipschitz with respect to time. Therefore, for any two time instants $t_{1} \leq t_{2}$, we have

$$
\begin{aligned}
\frac{e\left(t_{2}\right)}{m\left(t_{2}\right)}-\frac{e\left(t_{1}\right)}{m\left(t_{1}\right)} & =\frac{e\left(t_{2}\right)-e\left(t_{1}\right)}{m\left(t_{2}\right)}+\frac{e\left(t_{1}\right)}{m\left(t_{2}\right)}-\frac{e\left(t_{1}\right)}{m\left(t_{1}\right)} \\
& =\frac{\Delta e}{m\left(t_{2}\right)}-\frac{e\left(t_{1}\right)}{m\left(t_{1}\right)} \frac{\Delta m}{m\left(t_{2}\right)}
\end{aligned}
$$

where

$$
\begin{gathered}
\Delta e=e\left(t_{2}\right)-e\left(t_{1}\right) \\
\Delta m=m\left(t_{2}\right)-m\left(t_{1}\right) .
\end{gathered}
$$

Denoting

$$
\Delta t=t_{2}-t_{1}
$$

we have from (4), (5), and (60)

$$
\begin{aligned}
\left|\frac{\Delta e}{\Delta t}\right| & \leq \max _{t_{1}<t<t_{2}}|\dot{e}| \\
& \leq \max _{t_{1}<t<t_{2}}(k|x|+k) .
\end{aligned}
$$

From the above and (24) it follows that the first term in the RHS of (71) satisfies

$$
\left|\frac{\Delta e}{m\left(t_{2}\right)}\right| \leq \dot{k} \Delta t
$$

In view of the definition of $m$ in (24) we also have (see also $(1),(7)$, and (60))

$$
\begin{aligned}
\left|\frac{\Delta m}{\Delta t}\right| & \leq \max _{t_{1}<t<t_{2}}|\dot{x}| \\
& \leq \max _{t_{1}<t<t_{2}}(k|x|+k) .
\end{aligned}
$$

Using the above and (24) we obtain

$$
\left|\frac{\Delta m}{m\left(t_{2}\right)}\right| \leq k \Delta t
$$

since $e\left(t_{1}\right) / m\left(t_{1}\right)$ is bounded, introducing (76) and (78) into (71) we obtain

$$
\left|\frac{e\left(t_{2}\right)}{m\left(t_{2}\right)}-\frac{e\left(t_{1}\right)}{m\left(t_{1}\right)}\right| \leq k\left(t_{2}-t_{1}\right) .
$$

Since $e / m$ is $L_{2}$ and Lipschitz with respect to time it follows (see $[19$, p. 232]) that $e / m$ converges to zero. Boundedness of the state $x$ can then be shown by contradiction. Suppose that $x$ diverges, then, since $x_{m}$ is bounded, $x / m$ should converge to zero which is a contradiction (see (24)). In view of (58), all the signals remain bounded which concludes the proof.

\section{ConCLusions}

This paper has presented an adaptive control for a class of first-order nonlinear systems having the form $\dot{x}=c^{* T} f(x)$ $+b^{*} u$. The proposed control scheme does not require neither a priori knowledge of the sign of $b^{*}$ nor a priori knowledge on a lower bound on $\left|b^{*}\right|$. The control scheme is free from singularities, i.e., divisions by an estimate whose value can be close to zero. This has been possible by appropriately modifying the plant parameters estimates before using them in the control input law. On the other hand, since resulting control law presents discontinuities, existence of the solutions of the differential equations involved in the closed-loop control system has been established. The convergence analysis has finally shown that all the signals remain bounded and that the tracking error converge to zero.

\section{APPENDIX}

We show here that $P \leq P(0)$ implies that $\|P\| \leq\|P(0)\|$ where $\|P\|$ denotes the euclidean norm of $P$.

The proof will be based on the following facts (see for instance [20])

$$
\begin{gathered}
\exists L(n \times n): P=L L^{T} \\
\|P\|^{2}=\operatorname{tr}\left(P^{T} P\right)=\operatorname{tr}\left(P^{2}\right) \\
\operatorname{tr}(A B)=\operatorname{tr}(B A) \\
A-B>0 \Rightarrow \operatorname{tr} A-\operatorname{tr} B>0
\end{gathered}
$$

for any matrices $A(n \times m)$ and $B(m \times n)$.

Since $P \leq P(0)$

$$
\begin{aligned}
\|P\|^{2} & =\operatorname{tr}\left(L^{T} L L^{T} L\right) \leq \operatorname{tr}\left(L^{T} L(0) L(0)^{T} L\right) \\
& =\operatorname{tr}\left(L(0)^{T} L L^{T} L(0)\right) \\
& \leq \operatorname{tr}\left(L(0)^{T} L(0) L(0)^{T} L(0)\right) \\
& =\operatorname{tr}\left(L(0) L(0)^{T} L(0) L(0)^{T}\right) \\
& =\operatorname{tr}\left(P(0)^{2}\right) \\
& =\|P(0)\|^{2}
\end{aligned}
$$

\section{ACKNOWLEDGMENT}

The authors are grateful to Prof. A. S. Morse for his valuable comments on the paper.

\section{REFERENCES}

[1] A. S. Morse, "Global stability of parameter-adaptive control systems," IEEE Trans. Automat. Contr., vol. AC-25, no. 3, pp. $433-439,1980$.

[2] K. S. Narendra, Y.-H. Lin, and L. S. Valavani, "Stable adaptive controller design-Part II: Proof of stability," IEEE Trans. Automat. Contr., vol. AC-25, no. 3, pp. 440-448, 1980. 
[3] P. A. Ioannou and K. Tsakalis, "A robust direct adaptive controller," IEEE Trans. Automat. Contr., vol. 31, no. 11, pp. 1033-1043, 1986.

[4] D. G. Taylor, P. V. Kokotovic, R. Marino, and I. Kanellakopoulos, "Adaptive regulation of nonlinear systems with unmodeled dynamics," IEEE Trans. Automat. Contr., vol. 34, no. 4, pp. 405-412, Apr. 1989.

[5] S. S. Sastry and A. Isidori, "Adaptive control of linearizable systems," IEEE Trans. Automat. Contr., vol. 34, no. 11, pp. 1123-1131, Nov. 1989.

[6] B. Martensson, "Remarks on adaptive stabilization of first-order nonlinear systems," Syst. Contr. Lett., vol. 14, pp. 1-7, 1990.

[7] A. S. Morse, "Recent problems in parameter adaptive control," in Outils et Modèles Mathématiques pour l'Automatique, l'Analyse de Systèmes et le Traitement du Signal, I. D. Landau, Ed. Editions du CNRS, vol. 3, pp. 733-740, 1983.

[8] R. D. Nussbaum, "Some remarks on a conjecture in parameter adaptive control," Syst. Contr. Lett., vol. 3, no. 5, pp. 243-246, 1983.

[9] D. R. Mudgett and A. S. Morse, "Adaptive stabilization of linear systems with unknown high frequency gains," IEEE Trans. $\mathrm{Au}$ tomat. Contr., vol. AC-30, pp. 549-554, June 1985

[10] R. Lozano, J. Collado, and S. Mondié. "Model reference adaptive control without a priori knowledge of the high-frequency gain," IEEE Trans. Automat. Contr., vol. 35, no, 1, pp. 71-78, Jan. 1990

[11] R. Lozano and J. Collado, "Adaptive control for systems with bounded disturbances," IEEE Trans. Automat. Contr., vol. 34, no. 2, pp. $225-228$, Feb. 1989

[12] R. H. Middleton and P. V. Kokotovic, "Boundedness properties of simple indirect adaptive control systems," Tech. Rep. submitted to 1991 Amer. Contr. Conf.; also IEEE Trans. Automat. Contr., submitted for publication.

[13] J. M. Krause and P. P. Khargonekar, "Parameter information content of measurable signals in direct adaptive control," IEEE Trans. Automat. Contr., vol. AC-32, no. 9, Sept. 1987.

[14] S. C. A. Thomopoulos and Y. N. M. Papadakis, "On the existence of solutions in adaptive control" in Proc. 29th IEEE Conf. Decision Contr., Honolulu, HI, Dec. 1990.

[15] R. Lozano and B. Brogliato, "Adaptive control of a class of first order nonlinear systems without a priori information on the plant parameters," in Proc. 29th IEEE Conf. Decision Contr., Honolulu, HI, Dec. 1990.

[16] R. Lozano, J. M. Dion, and L. Dugard, "Singularity-free adaptive pole placement using periodic controllers," in Proc. 29th IEEE Conf. Decision Contr., Honolulu, HI, Dec. 1990.
[17] E. A. Coddington and N. Levinson, Theory of Ordinary Differential Equations. New York: McGraw-Hill, 1955.

[18] S. Sastry and M. Bodson, Adaptive Control Stability, Convergence and Robustness. Englewood Cliffs, NJ: Prentice-Hall, 1989

[19] C. A. Desoer and M. Vidyasagar, Feedback Systems: Input Output Properties. New York: Academic, 1975.

[20] P. Lancaster and M. Tismenetsky, The Theory of Matrices. New York: Academic, 1985

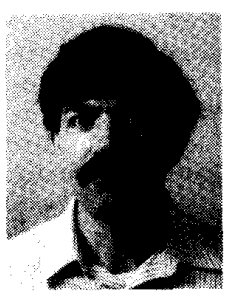

Rogelio Lozano was born in Monterrey, México, on July 12,1954 . He received the B.S. degree in electronic engineering from the National Polytechnic Institute of México in 1975, the M.S. degree in electrical engineering from Centro de Investigacion y de Estudios Avanzados (CIEA), México, in 1977 and the Ph.D. degree in automatic control from Laboratoire d'Automatique de Grenoble, France, in 1981 .

He joined the CIEA, México, in 1981 and was head of the Section on Automatic Control from 1985 to 1987. He spent a one-year study leave at the University of Newcastle, Australia, from 1983 to 1984 and was a Senior Research Associate at NASA Langley Research Center, VA, from 1987 to 1988 . He was a Visiting Professor at Laboratoire d'Automatique de Grenoble from March 1989 to July 1990. Since October 1990 he has been CNRS Research Director at Université de Technologie de Compiègne, France.

Dr. Lozano is presently Associated Editor of Automatica.

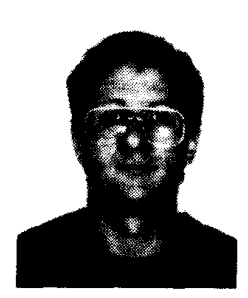

Bernard Brogliato was born in Saint Symphorien de Lay on April 11, 1963. He graduated from the Ecole Normale Supèrieure de Cachan Mechanical and Industrial Engineering Department, Paris, France (Sept. 1983-July 1987). Once his military obligations were fulfilled, he then "switched" to automatic control and joined the Laboratoire d'Automatique de Grenoble, Institut National Polytech nique de Grenoble, in September 1988. He received the $\mathrm{Ph} . \mathrm{D}$. degree in automatic control from the I.N.P.G. in January 1991. Since October 1991 he has been Chargé de Recherches at C.N.R.S. in the same laboratory. His main research interests are robots control and adaptive control. 